\title{
Gestión y educación financiera: clave para el manejo eficiente de las finanzas personales y empresariales
} Financial management and education: key to the efficient management of personal and business
finances.

* Claudia B. Lechuga Canto ${ }^{a}$, Beatriz Sauza Ávila ${ }^{b}$, Suly S. Pérez Castañeda ${ }^{c}$, Dorie Cruz-Ramírez $^{d}$

\begin{abstract}
:
Personal finances are a fundamental part of the economic activity of individuals, and to strengthen economic exchange and well-being requires the use of tools that allow sound financial decision-making in the short, medium and long term. In this sense, it is important to maintain healthy finances from a personal, family and business level, having clear knowledge about the efficient management of money and the economic resources that society has.
\end{abstract}

\section{Keywords:}

Finance, resources, money, income and expenses, education and financial management

\section{Resumen:}

Las finanzas personales son parte fundamental para la actividad económica de los individuos, y para fortalecer el intercambio y bienestar económico se require el manejo de herramientas que permitan la toma de decisiones financieras sanas en el corto, mediano y largo plazo. En este sentido es importante mantener finanzas sanas desde el ambito personal, familiar y empresarial, teniendo conocimientos claros sobre el manejo eficiente del dinero y de los recursos económicos que posee la sociedad.

\section{Palabras Clave:}

Finanzas, recursos, dinero, ingresos y gastos, educación y gestión financiera.

\section{Introducción}

La presente investigación surge por la necesidad de identificar la importancia que tiene el manejo financiero en los jóvenes estudiantes del Programa Educativo de Contaduría de la Escuela Superior de Ciudad Sahagún, para ello se realizó una encuesta a 58 estudiantes con la finalidad de conocer el manejo financiero de sus recursos económicos, aplicando la encuesta a estudiantes que cursaron los semestres de quinto, sexto, séptimo, octavo y noveno semestre ya que han cursado asignaturas relacionadas con las finanzas.

\footnotetext{
a Autor de Correspondencia, Universidad Autónoma del Estado de Hidalgo, Escuela Superior de Ciudad Sahagún, Email: claublc@uaeh.edu.mx

${ }^{\mathrm{b}}$ Universidad Autónoma del Estado de Hidalgo, Escuela Superior de Ciudad Sahagún, https://orcid.org/0000-0002-7919-6792, Email: beatriz_sauza@uaeh.edu.mx

${ }^{c}$ Universidad Autónoma del Estado de Hidalgo, Escuela Superior de Ciudad Sahagún, https://orcid.org/0000-0002-3763-9233, Email: sulysp@uaeh.edu.mx

${ }^{d}$ Universidad Autónoma del Estado de Hidalgo, Escuela Superior de Ciudad Sahagún, https://orcid.org/0000-0002-7853-7655, Email: doriec@uaeh.edu.mx
} 


\section{Importancia de las finanzas}

Al hablar de la importancia que tienen las finanzas es necesario identificar las características de la educación financiera, de la relevancia que tiene para la población, del diagnóstico sobre la situación financiera que tienen los individuos de cierta region con el proposito de realizar un plan de acción financiera que permita unas finanzas sanas desde el ámbito personal, familiar y empresarial.

En este sentido, se entiende que las finanzas conforman un área de la economía que apoya la administración eficiente de los recursos y es útil para la adecuada toma de decisiones y el cumplimiento de objetivos y metas planeadas.

Las finanzas personales tienen relación con las instituciones financieras, con las compras y los gastos que realizamos y conllevan a la elaboración de un presupuesto que permita organizar y controlar el uso de los recursos que se poseen, identificando los ingresos fijos, gastos fijos y gastos hormiga que generan desequilibrio financiero $y$ endeudamiento. 2

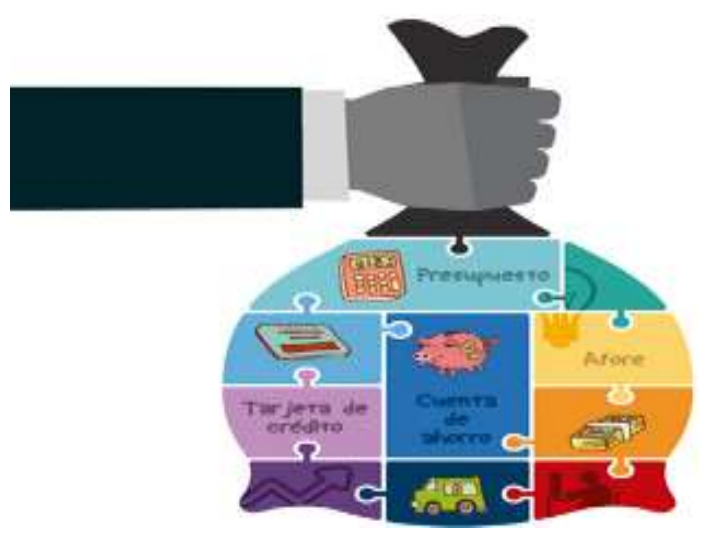

\section{Figura 1: CONDUSEF}

Para determinar si las finanzas son sanas, se debe considerer que los ingresos sean superiores a los gastos, esto genera ahorro, mismo que a futuro se traduce en inversion. $Y$ si los gastos son mayores que los ingresos se rebasa la capacidad de ahorro y se recurre al endeudamiento y financiamiento. Dicha situación se puede controlar identificando los gastos innecesarios y reflexionando si dichos desembolsos son necesarios 0 no.3

\section{Gestión financiera: visión en las finanzas personales, familiares y empresariales.}

La gestión y planeación financiera es un tema que hoy en día posee mayor importancia porque las personas realizan mayores compras generándose más gastos en sus finanzas con niveles de endeudamiento altos que impiden una economía personal sana.

Al hablar de gestión financiera se debe tener en mente como resolver el problema de liquidez y rentabilidad, tratando de proveer los recursos posibles que conlleven a la toma de decisiones más eficiente y garanticen certidumbre a las empresas, para lo cual se requiere tener una visión que se debe sustentar en el manejo de las finanzas personales y familiares. 1

Desde el punto de vista de las finanzas personales es importante plantearse ¿cuál es el exito financiero que se pretende alcanzar?, y para ello se debe reflexionar primeramente en que sueños y metas deseo lograr y posteriormente preguntarse con que fortuna pretendo alcanzarlo. Razón por la cual se deben plantear metas no financieras, las cuales pueden asociarse a las familiares, educativas, religiosas, sociales, entre otras, y las metas financieras que permiten poseer una capacidad monetaria con la cual se cumpla con las metas no financieras. Además, se debe tomar en cuenta el periodo de tiempo (corto ó largo plazo) en el cual se logra el cumplimiento de metas, y todo ello teniendo una relación con la planeación financiera de las personas con su nivel de ingresos y gastos. 6

\section{Educación Financiera}

La educación financiera así como la educación en general es uno de los grandes motores del desarrollo social y económico, al permitir la generación del desarrollo humano. De acuerdo con un estudio de la Comisión Nacional para la Protección y Defensa de los Usuarios de Servicios Financieros (CONDUSEF) el $62 \%$ de los mexicanos carece de educación financiera, el $80 \%$ de las familias ahorra fuera del sistema financiero y el $31 \%$ de los mexicanos gasta más de su nivel de ingreso; cifras que demuestran la carencia de educación y planeación financiera, a pesar de que distintas iniciativas del sector público, privado y social han empezado a observar un fuerte interés en éste ámbito, la cobertura de la educación financiera aún es limitada y el impacto no es claro. 4

La Comisión Nacional para la Protección y Defensa de los Usuarios de Servicios Financieros -CONDUSEF considera que la educación financiera es una aliada en el 
manejo de las finanzas, que tiene como propósito dotar a las personas de los conocimientos y habilidades necesarias para que en su vida cotidiana puedan realizar elecciones racionales sobre el uso y manejo apropiado de productos y servicios financieros. 5

Para Núñez (2013), la educación financiera es aquella que "transmite conocimiento, habilidades y actitudes necesarias para que la gente puede adoptar buenas prácticas de manejo del dinero para la generación de ingreso, gastos, ahorro, endeudamiento e inversión".7

Los elementos que integra la educación financiera, de acuerdo con la (Red Financiera 2008: citada por Rivera y Bernal, 2018; en Gómez, 2018) establece que este término, bajo un enfoque integral, contiene elementos como "el presupuesto de gastos, el ahorro, la inversión, el crédito y los seguros".8

\section{Los gastos que hacen los jóvenes}

Independientemente de la edad del jefe de hogar y de acuerdo con la Encuesta Nacional de Ingresos y Gasto de los Hogares (ENIGH) 2014 de INEGI, los principales destinos del ingreso se enfocan a cubrir el gasto en alimentos, bebidas y tabaco $(34.1 \%)$, transporte $(18.8 \%)$ y servicios de educación (14.0\%). En este sentido, el estudio de la UNAM y Banamex muestra la manera cómo los jóvenes distinguen gastos necesarios e innecesarios:

- Gastos necesarios: transporte, escuela de los hijos y útiles escolares, telefonía móvil, renta, alimentación, servicios (luz y agua).

- Gastos innecesarios: diversión y esparcimiento (ir a restaurantes, cines, centros nocturnos, casinos, fiestas) y cosas que les gustan, pero que "no son indispensables", como ropa, zapatos, aparatos electrónicos (teléfonos inteligentes, tabletas, televisores) o ir al gimnasio. 9

\section{Metodología de Investigación}

Para determinar cómo están las finanzas personales en un grupo de estudiantes de quinto a noveno semestre del Programa Educativo de Contaduría de la Escuela Superior de Ciudad Sahagún se llevó a cabo un estudio en el cual se estableció un modelo descriptivo, transversal y cualitativo; identificando la importancia que tiene la gestión y educación financiera con el manejo de la finanzas personales, transversal al realizar un levantamiento de campo en un momento específico en el tiempo, que fue abril de 2019 y cualitativo, porque la medición de sus conocimientos financieros se realizo partiendo de la percepción que poseen los estudiantes de estos temas, aplicando una encuesta con la finalidad de identificar el manejo de sus finanzas y si tienen una planeación financiera.

La muestra se efectuó por conveniencia, considerándose a los estudiantes que cursaban quinto, sexto, séptimo, octavo y noveno semestre, teniendo un total de 58 encuestados.

\section{Resultados}

Para identificar el manejo financiero que poseen los estudiantes de quinto a noveno semestre del Programa educativo de Contaduría se plantearon las siguientes preguntas:

\section{Gráfico. 1}

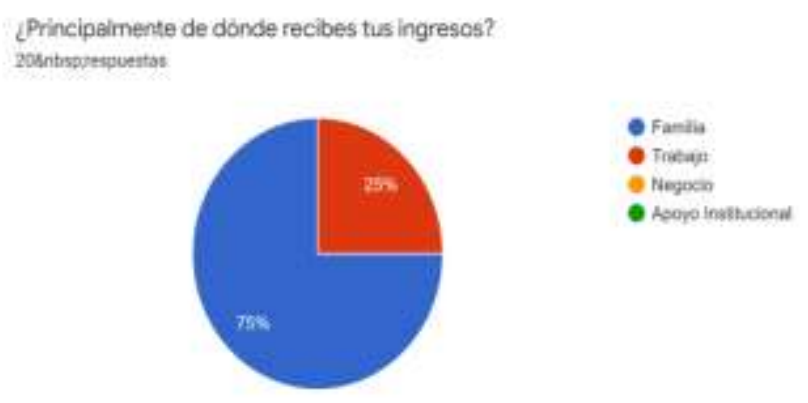

Fuente: Elaboración propia

En esta gráfica se observa que un alto porcentaje de los alumnos entrevistados afirman recibir dinero de su familia, representando el $75 \%$ y solo un $25 \%$ su principal fuente de ingreso es de un trabajo.

\section{Gráfico. 2}

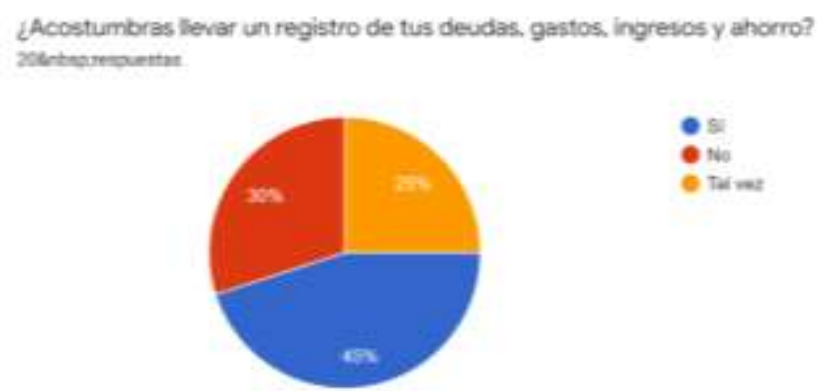

Fuente: Elaboración propia

El $45 \%$ de los alumnos encuestados afirmo llevar un registro de sus ingresos, ahorros y gastos mientras que un $30 \%$ no 
lleva ningún tipo de registro de sus gastos e ingresos y el resto algunas veces ha realizado un registro.

\section{Gráfico.3}

Elaboras un presupuesto personal para la distribución de tus ingresos zoenoapreaputatas
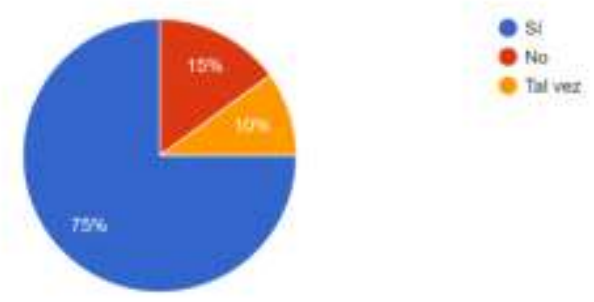

Fuente: Elaboración propia

En cuanto a este gráfico, del total de los alumnos encuestados el $75 \%$ lleva a cabo un presupuesto para la distribución de sus ingresos, mientras que el $15 \%$ no lleva un control de sus ingresos y el $10 \%$ restante algunas veces.

\section{Gráfico.4}

Pars ti z Oue ns ol ahorrot

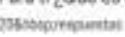
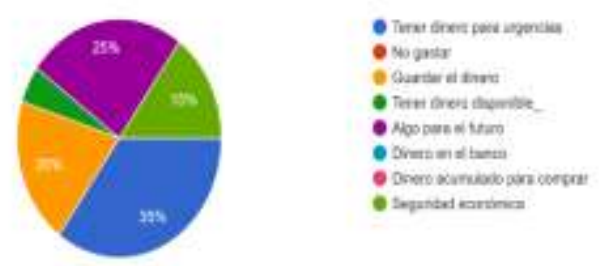

Fuente: Elaboración propia

Del total de alumnos entrevistados el 35\% dice que el ahorrar es tener dinero para urgencias, mientras que para el $25 \%$ es tener algo para el futuro, el $20 \%$ piensa que es guardar el dinero, el $15 \%$ para tener seguridad económica y el resto para tener el dinero disponible en el bolsillo.
¿Tu tienes el hábito de ahorrar?

zociteproquertie

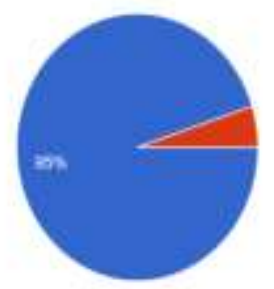

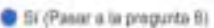
- Ne iPasar a la pongurna 17 )

Fuente: Elaboración propia

En este gráfico se observo que el $95 \%$ de los alumnos entrevistados tienen el hábito del ahorro.

\section{Gráfico.6}

\section{¿Que porcentaje de tus ingresos destinas al ahorro?}

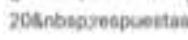

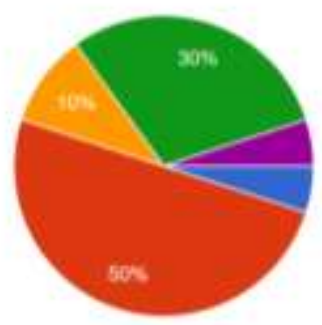

Fuente: Elaboración propia

El $50 \%$ de los alumnos encuestados destinan de sus ingresos un $10 \%$ al ahorro mientras que el $30 \%$ de los destina un $20 \%$ a su ahorro, lo cual se relaciona con la regla 50/30/20 para controlar los recursos y destinar el $20 \%$ al ahorro.

\section{Gráfico.7}

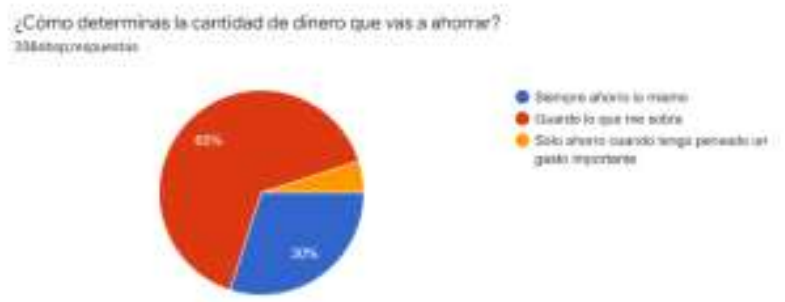

Fuente: Elaboración propia

Del total de alumnos entrevistados el $30 \%$ de ellos tiene un control de sus ahorros y un porcentaje fijo de ahorro, mientras que el $65 \%$ ahorra lo que le sobra y el $5 \%$ ahorra únicamente cuando se le presente un gasto importante.

\section{Gráfico.5}




\section{Gráfico.8}

A traves de que metbos acostumbras aharra?

A traves de que

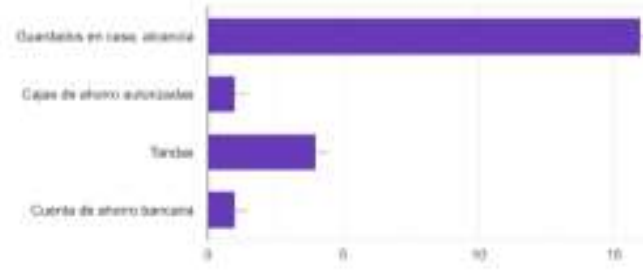

Fuente: Elaboración propia

La mayoría de los estudiantes acostumbran tener un ahorro guardado en su casa, como en alcancías, mientras que un porcentaje mínimo lo tiene ahorrado en cuentas de ahorro bancarias en instituciones formales.

\section{Gráfico.9}

\section{Cuales la racon por la que ti ahorres?}
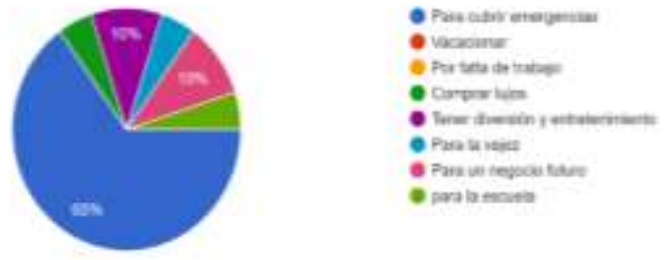

Fuente: Elaboración propia

El $65 \%$ de los alumnos entrevistados afirman ahorrar para cubrir emergencias, mientras que el $10 \%$ lo ahorran para gastarlo en diversión y entretenimiento, un $10 \%$ para un negocio futuro y el $15 \%$ restante se encuentra distribuido en la compra de lujos, en ahorrar para la vejez y en ahorrar para la escuela.

\section{Gráfico.10}

\section{¿Usas aigûn tipo de tarjeta bancaria?} 208ntosprescuesta!

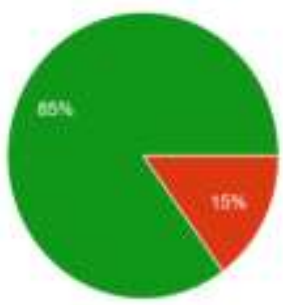

\section{Fuente: Elaboración propia}

Del total de alumnos entrevistados, cerca del $85 \%$ de los alumnos no cuentan con ningún tipo de tarjeta bancaria, unicamente el 15\% manejan tarjeta de débito bien por depositos de los padres, del pago de becas o bien por el pago del sueldo.

\section{Gráfico.11}

Cus et pars tiuna inverión?
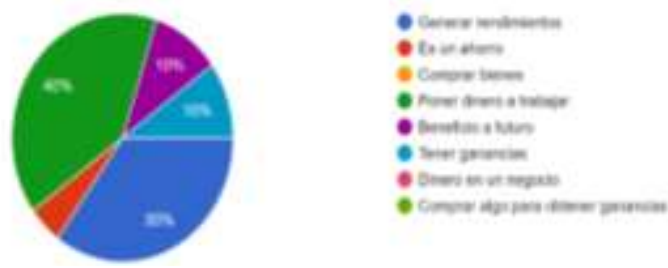

Fuente: Elaboración propia

Para el $40 \%$ de los alumnos entrevistados una inversión es poner el dinero a trabajar, para un $30 \%$ la inversión la ven mas como generar rendimientos y un $10 \%$ lo ven como beneficios a fututo, esto es un factor clave que explica el nivel de conocimientos financieros.

\section{Gráfico.12}

\section{¿Has invertido alguna vez? \\ 20sinbeprespoestas}
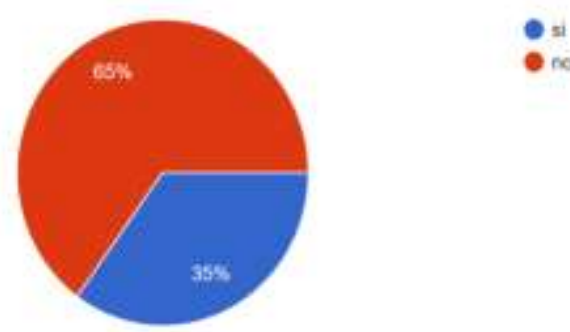

en

Fuente: Elaboración propia

De lo alumnos entrevistados el $65 \%$ afirma nunca haber invertido esto debido a tener escasos conocimientos sobre educación financiera y al temor a perder su dinero. 
Gráfico.13

Para tiel credito es:

20lentappoescentus
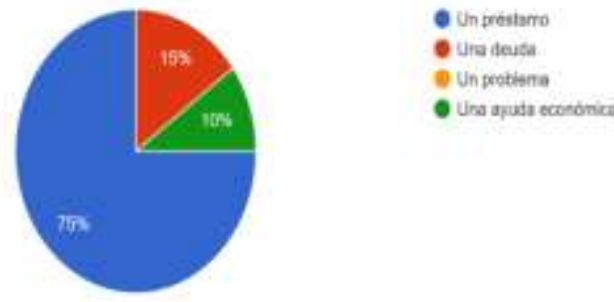

Fuente: Elaboración propia

Para el $75 \%$ de los estudiantes entrevistados un crédito es un préstamo y para el $15 \%$ s una deuda, mientras que para un $10 \%$ es una ayuda económica.

\section{Gráfico.14}

En caso de necesitarlo iA quièn le pedirias un crédito?

zosntappsspuestis
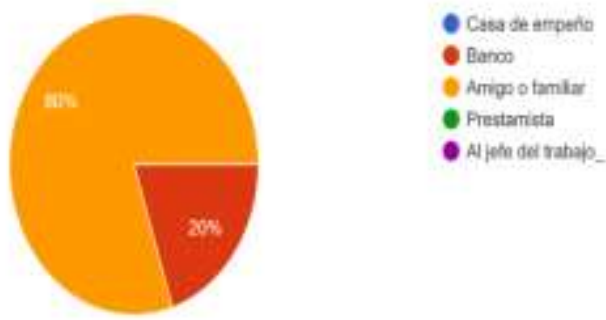

Fuente: Elaboración propia

El $80 \%$ de os alumnos le pedirían un crédito a un amigo o a un familiar, mientras que solo un $10 \%$ se lo pediría a una institución financiera formal

\section{Gráfico.15}

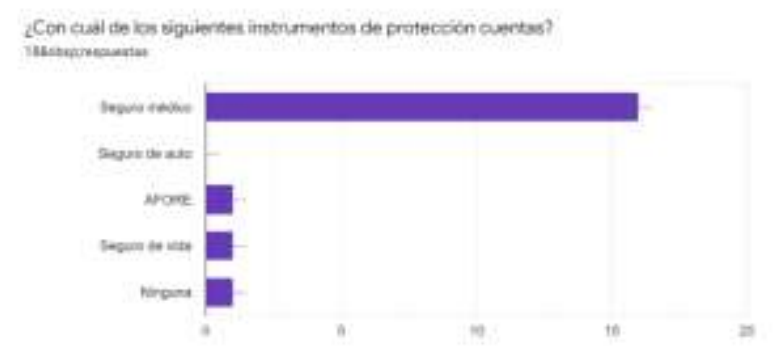

Fuente: Elaboración propia

El $75 \%$ de los alumnos entrevistados cuentan con un seguro médico vigente para la protección de su salud, mientras que el $10 \%$ cuenta con un seguro de vida y otro $10 \%$ cuenta con
AFORE y un $5 \%$ no cuenta con ningún instrumento de protección.

\section{Gráfico.16}

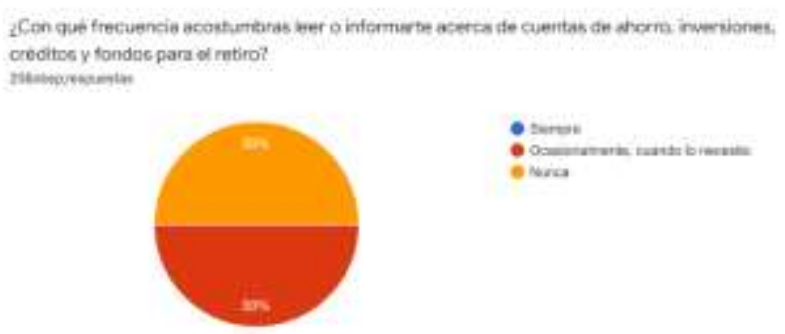

Fuente: Elaboración propia

El $50 \%$ del total de los entrevistados ocasionalmente leen información que tenga que ver con cuentas de ahorro, inversiones, créditos y fondos para el retiro, mientras que el resto nunca acostumbra informarse sobre el tema.

\section{Conclusiones}

Con base en los resultados arrojados a través de la encuesta aplicada a los estudiantes de Contaduría se concluye que la investigación cumple con el objetivo al realizar el diagnóstico del manejo financiero sobre los recursos económicos con los que cuentan, los conocimientos que tienen sobre los elementos que integra la educación financiera, según la Red Financiera en México. Analizándose 58 encuestas de estudiantes de quinto a noveno semestre.

De acuerdo con los resultados obtenidos, se concluye que el $45 \%$ de los estudiantes encuestados de la Licenciatura en Contaduría si tienen conocimientos sobre el manejo de sus finanzas, teniendo claro un registro para administrar sus gastos, ingresos, deudas y ahorro mediante un presupuesto, y en ese sentido el 50\% ahorra el $10 \%$ de su ingreso sobre todo para destinarlo a emergencias e imprevistos, aunque lo guardan en casa y no en cuentas bancarias.

Otro aspecto importante de resaltar es que únicamente el $10 \%$ ahorra para gastos innecesarios como la diversión y el entretenimiento.

Finalmente, en cuanto a la inversión, el crédito y los seguros los estudiantes poseen escasos conocimientos sobre lo que son, y la consulta para informarse sobre estos elementos que forman parte de la educación financiera es ocasional, situación que podría generar riesgo e incertidumbre en el manejo financiero por no tener conocimiento pleno y detallado de las instituciones financieras que les brindan mayores beneficios en cómo 
invertir y obtener los mejores rendimientos, así como adquirir créditos a tasas de interés bajas y como proteger su dinero.

\section{Referencias}

[1] Terrazas Pastor (2009), Modelo de gestión financiera para una organización, perspectivas No.23, Universidad Católica Boliviana.

[2] Olmedo Figueroa D.(2019) Las finanzas personales, Revista de la escuela de administración de negocios No. 65, Universidad Bogotá Colombia.

[3] CONDUSEF (2017) Economia joven, Lo básico de tus finanzas personales.

[4] Ruiz Pérez (2010) Educación financiera en México. Recuperado el 25 de marzo de 2020 de:

http://ford.ciesas.edu.mx/downloads/2do_1_04.pdf

[5] CONDUSEF (2014). La Educación Financiera. Recuperado el 07 de marzo de 2020,

https://www.condusef.gob.mx/Revista/index.php/usuario-

inteligente/educacion-financiera/225-la-educacion-financiera.

[6] CONDUSEF (2014). La importancia de llevar un presupuesto. Recuperado de http://www.condusef.gob.mx/index.php/comunicadosde-prensa/960-la-importancia-de-llevar-un-presupuesto-snef

[7] Núñez, L. (2013). ¿Qué es la educación financiera? Instituto Mexicano de Contadores Públicos: México.

[8] Gómez M. (2018) La educación financiera en México, cuaderno de investigación no. 53, dirección general de análisis legislativo. Recuperado el 25 de marzo de 2020 de: http://bibliodigitalibd.senado.gob.mx/bitstream/handle/123456789/419 0/CI_53.pdf?sequence=1\&isAllowed=y

[9] PROFECO (2017). Los jóvenes y las finanzas. Recuperado el 25 de marzo de 2020 de: https://www.gob.mx/profeco/documentos/losjovenes-y-las-finanzas?state=published 